\title{
La educomunicación en la documentación pública, privada y académica colombiana en su comprensión de la cuestión social
}

\author{
Media Literacy in public, private and academic Colombian documentation in its \\ understanding of the social issue
}

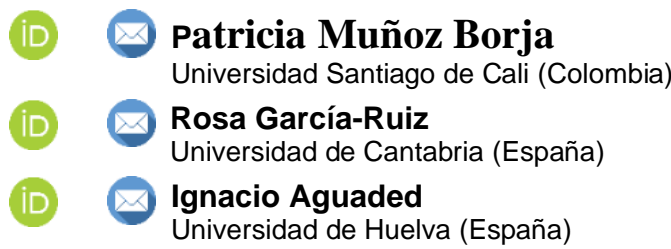

\section{Resumen}

This research proposed identify the policy actions, programs and projects for groups at risk of exclusion in Colombia and the roll of de media literacy in this context. Was performed a retrospective and descriptive bibliometric study that included documents from national and regional governmental and non-governmental agencies, specialized scientific publications, and, other informative publications about media literacy experiences. The results showed 1) a paradox about the comprehension and intervention to the population at risk of exclusion that was possible to observe to intertwine the characteristics of politics actions, programs and projects whit the characteristics of the vulnerable people or in risk of exclusion, 2) the media literacy in Colombia has a role very important because show two ways: is a strategy of education and is a mirror for to exhibit the paradox.

\section{Abstract}

This research proposed identify the policy actions, programs and projects for groups at risk of exclusion in Colombia and the roll of de media literacy in this context. Was performed a retrospective and descriptive bibliometric study that included documents from national and regional governmental and non-governmental agencies, specialized scientific publications, and, other informative publications about media literacy experiences. The results showed 1) a paradox about the comprehension and intervention to the population at risk of exclusion that was possible to observe to intertwine the characteristics of politics actions, programs and projects whit the characteristics of the vulnerable people or in risk of exclusion, 2) the media literacy in Colombia has a role very important because show two ways: is a strategy of education and is a mirror for to exhibit the paradox.

\section{Palabras clave / Keywords}

Educomunicación, población vulnerable, Colombia, actuaciones políticas, ciudadanía.

Media Literacy, vulnerable population, Colombia, policy actions, citizenship. 


\section{Introducción}

El propósito de esta investigación tuvo dos sentidos: identificar las actuaciones políticas, programas y proyectos dirigidos a los colectivos en riesgo de exclusión existentes en Colombia, e identificar el papel que la educomunicación juega en ese contexto, según las exigencias de formación en ciudadanía para fortalecer el pensamiento crítico y uso responsable y libre de los medios, que facilite la inclusión social. En Colombia los colectivos en riesgo de exclusión se denominan población vulnerable.

En las últimas décadas investigaciones sobre poblaciones vulnerables proliferan desde distintas perspectivas epistemológicas que, si bien permiten consolidar un corpus teórico y modelos de intervención social para el mejoramiento de la vida de dichos colectivos, siguen despertando interés debido a que aún no se han solucionado las situaciones de riesgo o superado las barreras.

Desde la educomunicación, garantizar el derecho a la información, la libertad de expresión o la libre comunicación en múltiples contextos es un reto, especialmente para colectivos vulnerables como niños o jóvenes (Aguaded, Caldeiro-Pedreira, \& Rodríguez-López, 2015), ancianos, mujeres o personas con discapacidad. Estos colectivos se ven afectados por la denominada brecha digital, que, según Lugo (2010), realmente es una brecha triple que involucra el distanciamiento tecnológico y digital entre países ricos y pobres, la desigualdad interna, y las expectativas de los más jóvenes respecto a los sistemas educativos.

A partir de lo anterior, se describirán algunas características de las personas pertenecientes a las poblaciones vulnerables, y, las características de las políticas, programas y proyectos dirigidos a dichas poblaciones. Concretamente se revisarán los conceptos de vulnerabilidad, resistencia, resiliencia, re-existencia, otredad y decolonialidad como características claves que han marcado históricamente los significados y prácticas sociales con los que dicha población ha construido su mundo y su subjetividad, y las características de las políticas, programas y proyectos dirigidos a esta población, entre las cuales se identificaron la discriminación positiva/acciones afirmativas, el enfoque diferencial y la participación social y ciudadana. Estas se tendrán en cuenta para comprender las propuestas nacionales de apoyo a la población vulnerable y la existencia de estrategias educomunicativas usadas en Colombia.

En los últimos 50 años se ha reflexionado sobre el pensamiento crítico, la pedagogía crítica y las mediaciones que muestran el aporte de la comunicación a la educación, tal y como proponían Freire $(1970 ; 1997 ; 2004)$, Martín Barbero (1991; 1996; 2002; 2014) o Kaplún (1985;1990; 1997;1998), como una necesidad imperiosa en Latinoamérica para facilitar transformaciones sociales. En este marco, la educomunicación se tomará como una estrategia pedagógica para la formación en ciudadanía crítica, como un medio que facilita la construcción de las subjetividades a partir del desarrollo de competencias para participar, opinar con conciencia crítica y crear colectivamente estrategias de transformación de la realidad.

La vulnerabilidad se relaciona con el riesgo de daño (ambiental, físico, emocional, económico, jurídico o social) que pueden sufrir las personas (Álvarez Múnera, 2010; Ullmann, Valera, \& Rico, 2014). Según la Comisión Económica para América Latina y el Caribe -CEPAL- (Pizarro, 2001) para identificar la vulnerabilidad se debe cruzar la "inseguridad e indefensión vivida a causa de un evento" con el "manejo de recursos y estrategias que usan las comunidades, familias y personas para enfrentar los efectos de ese evento". Por su parte Busso (2001), la define como "un proceso multidimensional" que une en una persona o grupo condiciones internas y externas que, al combinarse en un tiempo y espacio, deterioran sus condiciones de vida o su dignidad humana. En Colombia puede entenderse como la proponen Pizarro (2001) y Busso (2001) puesto que los habitantes, de las zonas rurales y urbanas, han sentido la inseguridad e indefensión provocadas por situaciones sociales, económicas y políticas reflejadas en el conflicto armado interno y sus consecuencias.

La resiliencia, resistencia y re-existencia en este contexto se entenderán como estrategias usadas por las personas para alejarse de la vulnerabilidad.

La resiliencia, según Becoña (2006) puede ser un proceso, un resultado, o, un proceso y resultado. Es un proceso cuando incluye mecanismos para cambiar el impacto de una situación de riesgo al que las personas logran adaptarse con éxito; es un resultado cuando las personas muestran la capacidad para mantener su funcionalidad afectiva y conductual a pesar de las adversidades; y es un proceso y resultado multifactorial cuando revisa los factores de riesgo y de protección del sujeto.

La resistencia incluye habilidades, actos o estrategias que unos utilizan al relacionarse con otros para sentar sus posiciones, evidenciar sus propias voces y mostrar que la relación de poder que se da entre ambos es bidireccional (Scott, 1985; 1986). Generalmente, aquellos que utilizan la resistencia son catalogados como débiles, marginales, excluidos o minoritarios por los que se auto-denominan fuertes, hegemónicos o mayoritarios. 
Los eventos históricos colombianos, muestran que las comunidades (indígenas, campesinas, afro, rurales y urbanas) han desarrollado competencias para mejorar su capacidad de resistencia ante las crisis (Tunubalá \& Pechené Muelas, 2010; Castillo, Camargo Abello, \& Morales, 2011; Pedraza, 2012; Gómez López, 2014; García Sánchez, 2015), y generalmente, involucran como elemento central del conflicto al territorio, cuyas representaciones simbólicas pueden considerar que su pérdida influye en la pérdida de su identidad (Tunubalá \& Pechené Muelas, 2010).

La re-existencia se refiere al proceso histórico y colectivo que los grupos étnicos han realizado para desaprender el proceso de colonización occidental y reconstruir su identidad desde sus prácticas cotidianas, sus sentidos de mundo, su relación con la naturaleza, con el planeta y con los otros, su cultura en general y su cosmovisión. La re-existencia implica formas de reconstrucción de los sujetos que tienen en cuenta su historia para visibilizar, reafirmar y revalorar lo que les es propio (Tunubalá \& Pechené Muelas, 2010; Albán Achinte, 2013).

Teniendo en cuenta lo anterior, la educomunicación para la formación ética y competencias ciudadanas y su repercusión en la construcción de la subjetividad en Colombia debe tener en cuenta al otro desde el estadío interpersonal, intercultural y de la praxis social (Teodoro Ramírez, 2007: 144), ya que rescatan a ese otro con su propia perspectiva de mundo (Ammann \& Da Porta, 2008; Walsh, 2015) y facilitan las relaciones sociales y sus procesos de interacción en los productos mediáticos que generan como prosumidores. Estos tres aspectos aluden principalmente a tres de las seis dimensiones de las competencias mediáticas (lenguajes, tecnología, interacción, producción y difusión, ideolología y valores y estética) identificadas por Ferrés y Piscitelli (2012): ideología y valores, lenguajes y procesos de interacción, ya que su apropiación dota al ciudadano de las competencias requeridas para consumir y producir información de calidad, libre y responsablemente.

Las actuaciones políticas, programas y proyectos, se caracterizan por incluir en la actualidad acciones afirmativas/discriminación positiva, el enfoque diferencial y la participación social y ciudadana, como respuestas a la historia colombiana en relación con la construcción de ciudadanía y el papel del Estado. En este trabajo se pretende precisamente identificar dichas actuaciones políticas, programas y proyectos dirigidas a las poblaciones vulnerables en Colombia y el papel de la educomunicación en este contexto en cuanto a la formación ciudadana para desarrollar pensamiento crítico y uso responsable y libre de los medios, que les facilite la inclusión en la sociedad actual.

\section{Metodología}

Para cumplir con el propósito, se desarrolló un estudio bibliométrico retrospectivo y descriptivo, que trianguló las fuentes utilizadas para garantizar su validez: material bibliográfico, documentos gubernamentales y no gubernamentales y experiencias educomunicativas colombianas dirigidas a poblaciones vulnerables. Dichas fuentes se organizaron, de manera manual, teniendo en cuenta dos marcos: cronológico (en las tres fuentes) y contextual (en las experiencias educomunicativas).

El corpus formado por 95 documentos en español, incluyó 36 documentos bibliográficos, 30 documentos gubernamentales y no gubernamentales, y 32 documentos de experiencias de educomunicación. Para seleccionar los 36 documentos bibliográficos se definió un marco cronológico que abarca desde los años 70 hasta los 90 , reuniendo a los autores clásicos de las temáticas centrales de la investigación, asumidas como dimensiones (educomunicación y vulnerabilidad) (Figura 1), y los autores que abordaron los mismos temas desde el año 2000 hasta 2018; los 30 documentos gubernamentales y no gubernamentales corresponden a políticas públicas o sociales, programas, proyectos, leyes o normativas institucionales de Colombia dirigidos a las poblaciones vulnerables, y otros internacionales, de la Comisión Económica para América Latina y el Caribe (CEPAL) y el Centro Internacional de Estudios Superiores de Comunicación para América Latina (CIESPAL); y los 32 documentos de experiencias educomunicativas colombianas dirigidas a poblaciones vulnerables.

Se seleccionaron dos categorías (Colectivos en riesgo de exclusión/ población vulnerable colombiana y actuaciones políticas, programas y proyectos gubernamentales y no gubernamentales dirigidos a dicha población) para observar las dimensiones, dentro de las cuales se reconocieron 6 categorías emergentes 0 características que responden al propósito de la investigación, de las cuales 3 corresponden a características que los documentos gubernamentales y no gubernamentales dan a las poblaciones para las cuales fueron diseñados: resiliencia, resistencia y re-existencia, y 3 corresponden a las actuaciones políticas, programas y proyectos gubernamentales y no gubernamentales dirigidos a dicha población: acciones afirmativas/discriminación positiva, enfoque diferencial y participación social y ciudadana (Figura 1). 


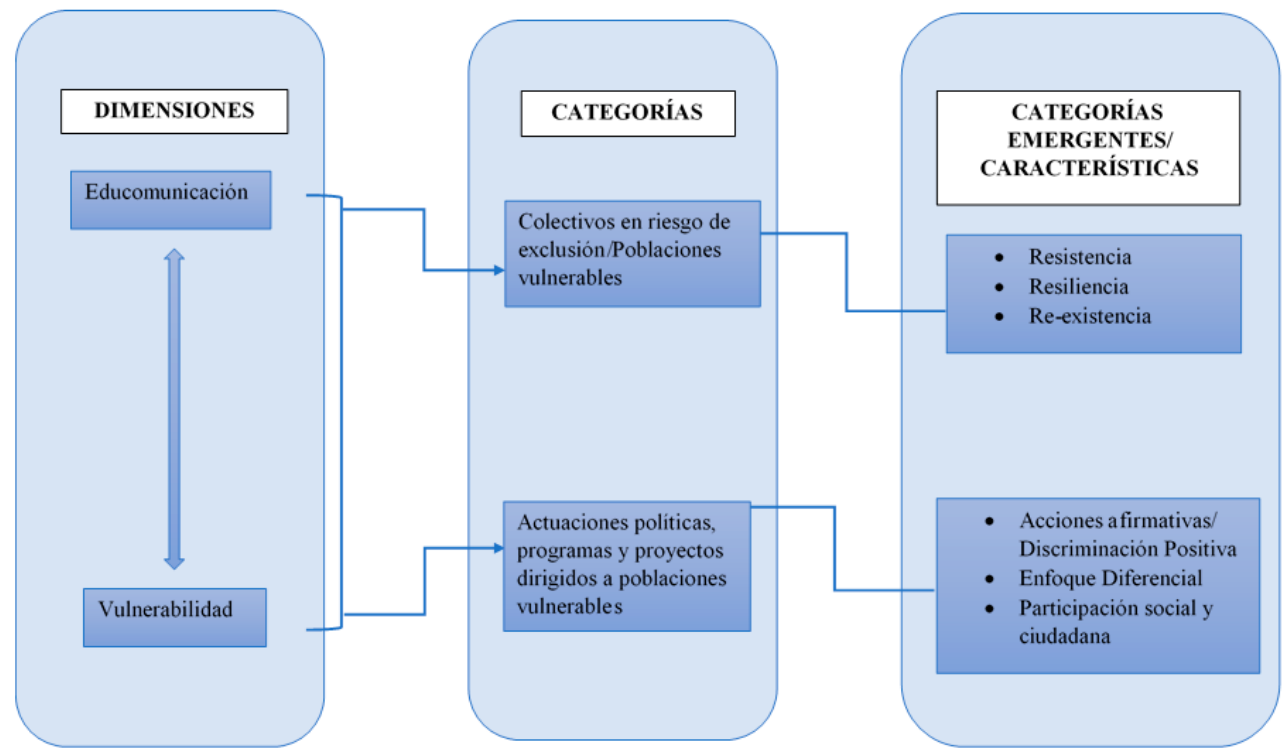

Figura 1. Dimensiones, Categorías y Categorías emergentes/Características definidas en la metodología

Se utilizó el análisis narrativo como "esquema cognitivo" para percibir e interpretar el mundo (Conde Gutiérrez, 2002; Chaves, Batista, \&Duarte, 2007), donde se identificaron las 6 categorías emergentes mencionadas y otros términos relacionados con las anteriores: competencia mediática, tecnologías de la información y la comunicación, educación, riesgo, vulneración, MOOC, oprimido, exclusión, amenaza, prosumidor, televisión educativa y radio comunitaria. Esta categorización mixta enriqueció la interpretación de los datos.

\section{Resultados}

\subsection{Frecuencia de aparición y evolución de los términos focales}

Al revisar los 95 documentos seleccionados, se comprobó que las categorías identificadas aparecen en la mayoría de los documentos fuente (Tabla 1). Existe mayor frecuencia de aparición en los documentos bibliográficos, aunque se evidencia la existencia de una serie de constantes terminológicas vinculadas a las categorías de análisis que se recogen en las diferentes fuentes documentales, poniendo el foco de atención en un rango temporal desde la década de los 70 hasta la actualidad.

\section{Tabla 1}

Frecuencia de aparición de categorías en los documentos

\begin{tabular}{|l|c|c|c|}
\hline \multicolumn{1}{|c|}{ Dimensiones/ Categorías } & $\begin{array}{c}\text { Documentos } \\
\text { gubernamentales y no } \\
\text { Documentos bibliográficos: } \\
\text { Libros o artículos de } \\
\text { revistas }\end{array}$ & $\begin{array}{c}\text { Documentos de } \\
\text { experiencias en } \\
\text { públicas o sociales, leyes, } \\
\text { resoluciones, acuerdos y } \\
\text { páginas web Estatales que } \\
\text { describen programas y } \\
\text { proyectos }\end{array}$ & $\begin{array}{c}\text { educomunicación: artículos } \\
\text { de revista, artículo de } \\
\text { periódico y páginas web de } \\
\text { organizaciones } \\
\text { gubernamentales y no } \\
\text { gubernamentales }\end{array}$ \\
\hline Vulnerabilidad & 713 & 266 & 48 \\
\hline Resiliencia & 307 & 7 & 11 \\
\hline Resistencia & 660 & 1 & 61 \\
\hline Re-existencia & 50 & & 1 \\
\hline $\begin{array}{l}\text { Educomunicación/Educación } \\
\text { mediática }\end{array}$ & 983 & 686 & 1357 \\
\hline TOTAL & $\mathbf{2 7 1 3}$ & $\mathbf{9 6 0}$ & $\mathbf{1 4 7 8}$ \\
\hline
\end{tabular}


Como se observa en la Tabla 1, el término re-existencia es más común en los documentos bibliográficos que en los gubernamentales y no gubernamentales o los que describen experiencias educomunicativas. Es un hallazgo esperado ya que dicho concepto se ha empezado a utilizar en la segunda década del siglo XXI para caracterizar a los pueblos latinoamericanos y colombianos que han estado excluidos, como los pueblos indígenas, afro y campesinos.

Por otro lado, el uso y apropiación de categorías como resistencia, resiliencia y re-existencia por parte del Estado o las experiencias de educomunicación, es menor a la que se encuentra en la literatura. Esto es comprensible puesto que la construcción teórica se realiza con mayor agilidad que la aplicación en la realidad (Muñoz-Borja, 2006: 84-86), ya sea en los lineamientos del Estado o en las experiencias cotidianas que usa la educomunicación como estrategia. Para completar el análisis de categorías respecto a la aparición de los términos focales conviene destacar que estos han evolucionado significativamente en el contexto de análisis, que existe la predominancia de algunos de ellos a lo largo de los años (p.e. el término oprimido se usó en 1970 por Freire y hoy sigue vigente) y que se evidencia una mayor y más reciente presencia de aquellos términos vinculados a la educomunicación.

El concepto educomunicación o educación mediática cuenta con mayor presencia en las dos primeras décadas del siglo XXI, junto con otros conceptos vinculados como competencias mediáticas, alfabetización mediática o tecnologías de la información y comunicación (TIC), con una frecuencia de aparición mayor a partir del 2007, principalmente en documentos bibliográficos. Asimismo, en documentos gubernamentales y no gubernamentales se recoge con una alta frecuencia el término TIC y televisión educativa. Respecto a los documentos vinculados a experiencias de educomunicación se recogen otros términos: prosumidor y MOOC; especialmente, a partir de la última década, u otros relacionados como radio comunitaria, o bachillerato por radio.

Por otro lado, al revisar la evolución de las relaciones establecidas entre las categorías de vulnerabilidad, resiliencia, resistencia y re-existencia, con la educomunicación, fue posible definir que, si bien 93 de los 95 documentos dan cuenta de la educomunicación en relación con las categorías mencionadas, algunos documentos solo establecían relación entre la educomunicación y una de las categorías, y la mayoría de los documentos establecían relación entre la educomunicación y dos o tres de las categorías, siendo más evidente con vulnerabilidad, seguida por resistencia, en tercer lugar, por resiliencia y, en cuarto, por reexistencia. Por ello, es posible afirmar que esta relación es directamente proporcional al surgimiento de los conceptos en la literatura científica.

Dichas relaciones en algunos documentos, según sus autores, están consolidadas y en otros están en proceso de apropiación (Tabla 2).

Los conceptos surgidos de esta búsqueda sistemática de términos relacionados con las categorías de análisis son numerosos, destacando respecto a la categoría vulnerabilidad: oprimido, riesgo, exclusión, amenaza, vulneración, exclusión y marginal, y se recogen todos desde 1991. Respecto a las categorías resiliencia y reexistencia no se han encontrado términos relacionados y en la categoría resistencia únicamente se halló el término decolonialidad con una frecuencia de aparición de 141 veces desde el 2008.

\section{Tabla 2}

Análisis relacional de las categorías de análisis con la educomunicación

\begin{tabular}{|l|r|r|}
\multicolumn{1}{|c|}{ Categorías } & En proceso & Educomunicación \\
\hline Vulnerabilidad & 10 & Consolidado \\
\hline Resiliencia & 3 & 51 \\
\hline Resistencia & 6 & 2 \\
\hline Re-existencia & 3 & 3 \\
\hline
\end{tabular}

De la Tabla 2, cabe destacar que las relaciones entre la educomunicación y las otras categorías vinculadas a las características de las poblaciones vulnerables muestran cómo el papel de la educomunicación se ha ido consolidando a través de los años como facilitadora de procesos de transformación social, política y cultural de los pueblos.

3.2. Características de las actuaciones políticas, programas y proyectos desarrollados en Colombia para los colectivos en riesgo de exclusión 
Esta segunda interpretación ofrece una exploración sobre 1) características de las actuaciones políticas, programas y proyectos colombianos desarrollados para beneficiar a las poblaciones vulnerables, y 2) características de estos colectivos relacionadas con las categorías identificadas inicialmente

Sobre las características de las actuaciones, los documentos gubernamentales tenidos en cuenta pertenecían a diferentes Ministerios y despachos del gobierno nacional y regional, y entidades no gubernamentales que prestan sus servicios a las poblaciones vulnerables del país. Entre estas se escogieron: la Constitución Colombiana de 1991, la Política Pública Nacional de Primera Infancia (Conpes-109, 2007), la Estrategia Integral de atención a la primera infancia "de cero a siempre" (Castro, Vizcaino, \& Alarcón Párraga, 2012; Contraloría General de la República de Colombia, 2014), la Política Pública de Primera infancia, infancia y adolescencia (ICBF \& PNUD, 2013), la Política Pública de Atención a la Discapacidad (Conpes-166, 2013), la Política Pública Nacional de Envejecimiento y Vejez (2007-2019) (Ministerio de la Protección Social, 2007), la Ley 581 por la cual se reglamenta "la adecuada y efectiva participación de la mujer en los niveles decisorios de las diferentes ramas y órganos del poder público, de conformidad con los artículos 13, 40 y 43 de la Constitución" (Congreso de Colombia, 2000), el Plan Decenal de Salud Pública -PDSP (2012 - 2021) (MSPS - PDSP, 2013), la Estrategia Familias en Acción (Prosperidad Social Colombia, 2016) y la estrategia de entrega de viviendas gratis a familias vulnerables (Urna de Cristal Gobierno de Colombia, 2013; ANSPE, 2014). Entre las propuestas de organizaciones no gubernamentales se seleccionaron Batuta, Fundación Carvajal, Fundación Ideal y Fundación Saldarriaga Concha, entre otras.

Las actuaciones políticas, programas y proyectos gubernamentales y no gubernamentales revisados, coincidieron en incluir 3 características, asumidas como categorías emergentes: acciones afirmativas/discriminación positiva, el enfoque diferencial y la participación social y ciudadana, como respuestas a la realidad de las poblaciones vulnerables.

La primera característica, acciones afirmativas/discriminación positiva, se utiliza como estrategia para facilitar la equiparación de oportunidades y la inclusión de la población vulnerable para mejorar sus condiciones de vida, beneficiando a aquellos que se han considerado socialmente en desventaja (Urteaga, 2009; Barrère Unzueta, 2003).

En Colombia, estas acciones se utilizan como estrategia de equiparación de oportunidades y de equidad para responder a las condiciones económicas, sociales y políticas del país, distribuir los recursos y ofrecer a los ciudadanos desde el Estado la oportunidad de mejorar sus condiciones de vida y facilitar el desarrollo y bienestar (Asamblea Nacional Constituyente, 1991); como ejemplos se pueden mencionar:

- el programa que enseña música a niños de bajos recursos económicos, escogiendo a los mejores talentos para conformar sus orquestas infantiles e impulsando a los mejores para continuar su formación (Fundación Nacional Batuta, 2017).

- el programa denominado 100.000 viviendas gratis, entrega viviendas a personas víctimas y actores del conflicto colombiano (Urna de Cristal Gobierno de Colombia, 2013).

- los centros recreativos municipales (Alcaldía de Cali, 2017) que ofrecen opciones de ocupación del tiempo libre a poblaciones no pertenecientes a las élites.

Las acciones afirmativas como estrategia para facilitar la inclusión de poblaciones vulnerables tienen en cuenta la propuesta de Amartya Sen (1999), esperando que las personas puedan entrelazar sus capacidades con las oportunidades brindadas por la sociedad para escoger cómo vivir y ejercer su ciudadanía. Estas acciones han fortalecido el ejercicio ciudadano, mantenimiento y recuperación de la dignidad humana, obligando a conocer y respetar los derechos y deberes propios, y de los demás.

En este contexto diversos despachos gubernamentales del ámbito nacional, departamental y municipal utilizan estrategias educomunicativas para efectivizar sus relaciones con los ciudadanos al capacitar e informar sobre las diversas acciones afirmativas, sus beneficios y efectos, que redundan en la formación de competencias ciudadanas al abrir espacios de reflexión y crítica (Delgado-Ponce, 2015).

La segunda característica, enfoque diferencial, reconoce la existencia de poblaciones con cualidades particulares como género, edad, discapacidad u orientación sexual, para tomar "medidas integrales" de "ayuda humanitaria, atención, asistencia y reparación" (SNARIV, 2011). Las evidencias mostraron que en Colombia se adoptó dicho enfoque cuando en la práctica identificaron personas con varias condiciones de vulnerabilidad, que dificultaban su ubicación en algún grupo o duplicaban los apoyos para la misma persona (p.e. mujeres campesinas, cabeza de hogar, desplazadas con discapacidad). 
La participación social y ciudadana, tercera característica, aumentó su importancia en Colombia debido a las situaciones históricas que llevaron a la implementación de la Constitución de 1991. Los documentos gubernamentales y no gubernamentales, en su mayoría, incluyen esta característica para cumplir con lo establecido en la normatividad y aportar a la transformación de los modos de relación entre los colombianos y el Estado.

\subsection{Papel de la educomunicación en las actuaciones políticas, programas y proyectos}

La educomunicación actualmente facilita procesos de participación social en la medida que ofrece mayores oportunidades de inclusión y accesibilidad al eliminar algunos elementos de interacción directa y presencial como la temporalidad, espacialidad y mediación.

La educomunicación y las nuevas tecnologías en relación con la participación ciudadana, ofrecieron otros modos de relación entre Estado y ciudadanos, y entre los propios ciudadanos (AGESIS, 2012); en la medida que generaron oportunidades de aprendizaje sobre derechos ciudadanos, competencias mediáticas y desarrollo de pensamiento crítico frente al consumo y producción de contenidos.

Las instancias gubernamentales colombianas cuentan en la actualidad con varias estrategias y acciones pensadas para generar espacios de participación ciudadana accesibles, que iniciaron enseñando a los ciudadanos los derechos y oportunidades que tienen para acceder a servicios, no obstante, cada vez surgen más escenarios virtuales gestionados por el Estado que facilitan la participación y el ejercicio ciudadano más allá del acceso a los servicios (Ministerio de Salud y Protección Social, 2017; MinTIC, 2016a; MinCultura, 2013; SENA-MinTrabajo, 2013a; MinTIC, 2017; MinTIC, 2018a; MinTIC, 2018b).

Sobre las características de la población vulnerable, es posible decir que, en Colombia, dentro de esta categoría se encuentran las mujeres cabeza de hogar, mujeres gestantes, niños, adulto mayor, desplazados, personas LGTBI, personas con discapacidad, personas en condición de pobreza y minorías étnicas, explicitadas en el artículo 13 de la constitución de 1991(Asamblea Nacional Constituyente, 1991). A partir de ésta, Colombia como "Estado social de derecho" (Asamblea Nacional Constituyente, 1991: Artículo 1), implementó políticas sociales y públicas, leyes y normas; además, de planes, programas, proyectos, estrategias y acciones dirigidas a estas poblaciones.

Las estrategias de educomunicación usadas en Colombia para difundir y formar a la ciudadanía sobre dichas acciones, proyectos, programas, planes y políticas han usado la televisión pública y las páginas web de las entidades estatales. Los canales de televisión utilizados son el Canal del Congreso, Señal Colombia, Canal Uno y Canal Institucional, los cuales tienen cobertura nacional (Señal Colombia, 2017; Canal Uno, 2017; Canal Institucional \& RTVC, 2017). Las páginas web en su mayoría entregan información y abren espacios de formación y comunicación con el ciudadano y su énfasis está en informar sobre derechos, servicios y rutas de comunicación con el Estado. Entre estas páginas están las del Ministerio de las TIC (MinTIC, 2016b) , Ministerio de Salud y Protección Social (Ministerio de Salud y Protección Social, 2017), Ministerio de Educación (Ministerio de Educación Nacional de Colombia, 2017), el Instituto Colombiano de Bienestar Familiar (Instituto Colombiano de Bienestar Familiar - ICBF-, 2016; 2017), entre otros.

El Estado ha usado la televisión, desde su llegada al país en el año 1954 (López, 2012), como estrategia fuerte de formación en aspectos históricos, ecológicos, culturales, sociales, y de ciudadanía. Sin embargo, esta establece una relación unidireccional con la audiencia, aspecto que no, necesariamente, aporta a la ciudadanía capacidades críticas para el consumo y producción de información. El uso de páginas web y tecnologías para aproximarse a los ciudadanos abrió la oportunidad de establecer una relación bidireccional entre el Estado y los colombianos. Esto generó espacios interactivos de formación ciudadana y de conocimiento de las políticas, planes, programas, proyectos y acciones diseñados para atender las condiciones de vulnerabilidad (MinTIC, 2017; MEN, 2013).

Con respecto a la resiliencia, resistencia, y re-existencia como estrategias para alejarse de la vulnerabilidad, se identificó que estas son usadas cotidianamente por comunidades colombianas rurales y urbanas para continuar con sus vidas y darle sentido a su existencia.

En Colombia se ha utilizado la radio y la televisión, públicas y comunitarias, como estrategias mediáticas para fortalecer acciones y movilizaciones relacionadas con la resiliencia, resistencia y re-existencia desde la segunda mitad del siglo XX (Mata, 1993).

En este marco, la educación por radio y la televisión educativa fueron importantes para las comunidades. Por ejemplo, la Fundación Acción Cultural Popular brindó herramientas de formación académica, ciudadana y fortalecimiento de la identidad y liderazgo de muchos campesinos colombianos mediante las escuelas radiofónicas realizadas desde Radio Sutatenza (Fundación Acción Cultural Popular, 2013). A través del 
programa Bachillerato por radio y de televisión educativa, muchos colombianos accedieron a la educación formal y posterior ingresaron en la universidad (López, 2012; RTVC \& Señal-Memoria, 2015). En estas estrategias de educomunicación rescataron los conceptos de otredad/alteridad y decolonialidad, entre otros, para visibilizar diversas cosmovisiones, prácticas sociales, estilos de vida, subjetividades y formas válidas de habitar el mundo en Colombia.

Sobre la otredad, los documentos revisados mostraron la necesidad de construir acciones políticas, programas, proyectos y estrategias de educomunicación, teniendo en cuenta la diversidad y no la homogeneidad en la medida que Colombia se auto-reconoce como "pluriétnica" y "multicultural" (Asamblea Nacional Constituyente, 1991; Osorio Marulanda, 2011).

La decolonialidad, como reconstrucción del ser humano, se encontró que en Colombia existen estrategias que han utilizado la educomunicación para facilitar procesos de "re-humanización", entre ellas están las radios comunitarias de algunos pueblos indígenas quienes; además de las mingas, usan este medio como espacio de participación de sus autoridades, jóvenes y ancianos para generar programas culturales que fortalezcan su cosmovisión y su identidad (Rincón et al., 2007: 135). Otras experiencias evidencian la participación de campesinos utilizando la radio y la televisión comunitaria y la existencia de experiencias de jóvenes y niños que han encontrado otros rumbos en sus vidas, diferentes a las pandillas, al mostrar la cotidianidad, otras realidades y la vida de sus barrios en su portal web (Iniciativa de Red de Comunicación, 2004; Rincón et al., 2007: 113).

En este contexto es importante rescatar la influencia que CIESPAL (2016) ha ejercido en la consolidación de la escuela de pensamiento crítico comunicacional latinoamericano, el cual rescata el papel de la comunicación como herramienta fundamental de formación, emancipación y liberación. De este modo, la CIESPAL se ha convertido en un referente importante en Latinoamérica y el mundo para mostrar otras ideas y voces, diferentes a las hegemónicas, sobre las cotidianidades que viven, las subjetividades y las culturas originarias para el buen vivir.

\section{Discusión y conclusiones}

Mientras las actuaciones, políticas, programas y proyectos colombianos para atender a la población vulnerable se caracterizan por incluir acciones afirmativas/discriminación positiva, el enfoque diferencial y la participación social y ciudadana (Asamblea Nacional Constituyente, 1991; Conpes-109, 2007; Conpes-166, 2013; Ministerio de la Protección Social, 2007; Congreso de Colombia, 2000; Prosperidad Social Colombia, 2016), se identificó que las características de los colectivos en riesgo de exclusión resaltadas en los documentos son la vulnerabilidad, la resistencia, resiliencia, re-existencia, el reconocimiento del otro y la decolonialidad.

Las primeras características pretenden responder a las segundas para mejorar las condiciones de vida de las personas beneficiadas, sin embargo, se evidenció una paradoja entre ambos conjuntos: las características identificadas en las actuaciones políticas, programas y proyectos del país están enmarcadas dentro del modelo económico capitalista con la intención (según los textos revisados) de responder a las necesidades de la población vulnerable. La visión de este modelo capitalista es homogeneizante, y las características identificadas en los textos académicos, los documentos de las experiencias educomunicativas y algunos documentos oficiales de entidades gubernamentales y no gubernamentales (Asamblea Nacional Constituyente, 1991; Tunubalá \& Pechené Muelas, 2010; Albán Achinte, 2013; Pedraza Gómez, 2012), se ubican en otras lógicas surgidas para visibilizar, reconocer y atender las necesidades desde lo diverso y heterogéneo.

Históricamente el pueblo colombiano ha desarrollado estrategias como la resiliencia, la resistencia y la reexistencia para distanciarse de las condiciones de vulnerabilidad, a través de diversas formas de educación y difusión, entre las cuales se encuentran el establecimiento de interacciones directas y presenciales, y la educomunicación.

Las estrategias de educomunicación se han implementado a través de medios de comunicación tradicionales como la radio y la televisión públicas y comunitarias, la prensa (en menor proporción) y las nuevas tecnologías en la última década.

En Latinoamérica, los procesos de decolonización que las comunidades vulnerables y no hegemónicas han hecho en el continente para recuperar su voz e identidad, es común encontrar investigaciones que rescatan el valor del otro y la construcción de la identidad latinoamericana desde la diversidad. Los resultados indican que para diseñar estrategias de educomunicación que realmente repercutan en la audiencia para alcanzar la transformación social deseada es necesario incluir principios y valores éticos que rescaten la diversidad y la 
pluralidad en la formación ciudadana cuya esencia está puesta en la diferencia y no en la homogeneidad, coincidiendo con las propuesta de Teodoro Ramírez (2007), Ammann \& Da Porta (2008) y Walsh (2015).

Al contrastar este deber ser con las experiencias educomunicativas revisadas, se evidenció que algunas de ellas, incluyen valores, prácticas sociales y costumbres propias de las audiencias a quienes van dirigidas, aspectos que resaltan la diversidad del país y el fortalecimiento identitario de sus gentes. También se identificó que otras estrategias educomunicativas presentan dos posturas dependiendo de quiénes las generan, hallazgo que no coincidió con ninguno de los documentos bibliográficos incluidos en la revisión. Por un lado, la postura hegemónica occidental para la formación ciudadana en algunas propuestas del Estado y ONGs, las cuales enfatizan en la educación en derechos y deberes de la ciudadanía y en el establecimiento de canales para la expresión de quejas y reclamos relacionados con dichos derechos; por otro lado, el reconocimiento y exaltación de la diversidad, valores y principios que representan las poblaciones no hegemónicas como grupos organizados que rescatan su dignidad e identidad. Entre estas posturas se encontraron las propuestas de grupos étnicos, grupos contra el maltrato a la mujer, y grupos LGTBI, entre otros.

En las estrategias educomunicativas observadas, se evidenció su aporte al reconocimiento de la diversidad del país y su avance para formar como ciudadanos críticos y prosumidores a los grupos vulnerables (GarcíaRuiz, Ramírez-García, \& Rodríguez-Rosel, 2014; García-Galera \& Valdivia, 2014).

Esta construcción de ciudadanos críticos tiene en cuenta la propuesta hegemónica occidental de entidades como la UNESCO y la Comisión Europea, y, está matizada por las corrientes de pensamiento latinoamericano, surgidas desde la segunda mitad del siglo XX hasta la fecha.

Dichas corrientes de pensamiento muestran la posibilidad de dar cuenta del otro con sus complejidades y formas distintas de aprender y vivir el mundo en el que se encuentra, además de fortalecer el capital social y el tejido asociativo existente en el país, sobre todo de los sectores marginados, que abren espacios de participación y ejercicio ciudadano, capaces de rescatar las diferencias culturales y sociales en un mundo globalizado que necesita el reconocimiento de lo local como elemento primordial para la construcción y el fortalecimiento de la identidad (Morin et al., 2006; Álvarez Múnera, 2010; Congreso de Colombia, 2010; Culver \& Jacobson, 2012).

Esto vuelve a demostrar la vigencia que tienen las propuestas de Freire, Kaplún y Martín-Barbero, con respecto a la necesidad de una educomunicación capaz de facilitar el desarrollo de un pensamiento crítico para interpretar la realidad, para producir contenidos mediáticos significativos que se adapten a su forma de ver y entender su mundo, para crear conciencia sobre la importancia de darles voz no solo en los procesos de participación y toma de decisiones como ciudadanos (Aguaded, 2012), sino también en la puesta en escena de su idiosincrasia, cultura e identidad, para dignificar sus cosmovisiones, prácticas sociales y estilos de vida, como otras formas válidas de ejercer la ciudadanía.

A la vista de los resultados obtenidos y de la discusión con las aportaciones de otros expertos y organismos, podemos concluir que al revisar la frecuencia de aparición de los conceptos incluidos en este estudio se evidenció el cambio conceptual hecho en Colombia durante los últimos 70 años respecto a los ciudadanos y las formas estatales de responder a las actuaciones políticas, programas y proyectos que ponen en marcha. Este hecho implica la manera de concebirse y nombrarse a las personas, lo que genera estrategias de solución coherentes con dicha concepción; es decir, la palabra utilizada lleva a la acción en su misma línea y, aunque los términos son apropiados más ágilmente desde el ámbito académico, progresivamente, son asumidos por la sociedad mediante los procesos de intervención.

Por otro lado, cabe destacar que la paradoja encontrada entre las características de las actuaciones políticas, programas y proyectos, y las características resaltadas de la población vulnerable, muestra las tensiones de posturas conceptuales y pragmáticas sobre el ciudadano y su relación con el Estado colombiano que se hacen evidentes en las estrategias de educomunicación. Por un lado, se encuentran propuestas educomunicativas que, aunque han abierto espacios de participación, se desarrollan desde perspectivas implementadas por el pensamiento occidental y capitalista que tiende a homogeneizar a las poblaciones. Estas estrategias permiten la aproximación de algunas audiencias, sin embargo, no posibilitan el acercamiento a la totalidad de las poblaciones que conforman la diversidad del país.

Las conclusiones apuntan también a que existen propuestas educomunicativas que desarrollan posturas políticas de auto-reconocimiento y reivindicación de los valores, formas de vida y diversidad de la población colombiana. Cabe destacar que estas estrategias se construyen en su mayoría desde los grupos que tradicionalmente han sido marginados en el país, aunque en ocasiones también desde algunas instancias gubernamentales. 
Esto denota la transición por la cual atraviesa el país sobre la comprensión de sus ciudadanos y su relación con el Estado; es decir, en lo cotidiano permanecen y conviven las lógicas de comprensión de la ciudadanía de los modelos hegemónicos y los modelos emergentes que proponen el rescate, y el reconocimiento de lo no hegemónico como parte integral de ese Estado.

La educomunicación en Colombia, además de ser facilitadora en procesos de formación sirve para evidenciar las tensiones existentes y los procesos de transición relacionados con las lógicas como se asume la ciudadanía y se definen las relaciones entre ciudadanos y Estado, por parte de los actores gubernamentales, los actores no gubernamentales y la comunidad en general que pertenece a diversos grupos vulnerables.

Para futuras investigaciones sería necesario ampliar la búsqueda de estrategias desarrolladas en otros países latinoamericanos o de otras latitudes que pudiesen replicarse y mejorar la situación colombiana. Así mismo sería conveniente contrastar los resultados encontrados con trabajos de campo que aporten información desde los mismos colectivos vulnerables, lo cual ya se ha empezado a desarrollar.

\section{Apoyos}

I+D " YOUTUBERS E INSTAGRAMMERS: LA COMPETENCIA MEDIATICA EN LOS PROSUMIDORES EMERGENTES" (RTI2018-093303-B-I00), financiados por el Fondo Europeo de Desarrollo Regional (FEDER) y la Agencia Estatal de Investigación del Ministerio de Ciencia, Innovación y Universidades de España.

\section{Referencias}

AGESIS. (2012). e-Participación. Conceptos básicos y buenas prácticas. Montevideo: AGESIC - Gobierno Abierto Eds. Recuperado de https://bit.ly/1cZvmLQ

Aguaded, I. (2012). Apuesta de la ONU por una educación y alfabetización mediáticas. Comunicar, 19(38), 7-8. http://dx.doi.org/10.3916/C38-2012-01-01 Apuesta

Aguaded, I., Caldeiro-Pedreira, M. C., \& Rodríguez-López, J. (2015). ¿Qué nos muestran las pantallas?: La mirada crítica adolescente en el marco de las industrias culturales y del pensamiento actual. Alteridad. Revista de Educación, 10(1), 8-20. https://doi.org/10.17163/alt.v10n1.2015.01

Albán Achinte, A. (2013). Pedagogías de la Re-existencia. Artistas indígenas y afrocolombianos. En Pedagogías decoloniales: Prácticas insurgentes de resistir, (re)existir y (re)vivir. TOMO I (Serie Pens). Quito, Ecuador. Alcaldía de Cali. (2017). Página web Centros Recreativos Municipales. Recuperado de https://bit.ly/2Xvlb2N Álvarez Múnera, J. R. (2010). Significados, categorías de análisis y posibilidades interpretativas del concepto de vulnerabilidad. Revista de La Facultad de Trabajo Social UPB, 26(26), 142-159.

Ammann, B., \& Da Porta, E. (2008). Rutas alternativas de la Comunicación. Procesos de significación social, ideología y poder. (Ferreyra, Ed.) (Ferreyra). Buenos Aires: Ferreyra. Recuperado de https://bit.ly/2XgUtXo

ANSPE. (2014). Agencia Nacional para la Superación de la Pobreza Extrema - ANSPE. Recuperado de https://bit.ly/2xbRwwV

Asamblea Nacional Constituyente. Constitución de 1991, Diario Oficial (1991). Colombia. Recuperado de https://bit.ly/2IJWo2B

Barrère Unzueta, M. Á. (2003). Igualdad y "Discriminación Positiva”: Un Esbozo de Análisis Teórico-Conceptual. Cuadernos Electrónicos de Filosofía del Derecho. España. Recuperado de https://bit.ly/2LfJbjS

Becoña, E. (2006). Resiliencia: Definición, características y utilidad del concepto. Revista de Psicopatología y Psicología Clínica, 11(3), 125-146. Recuperado de https://bit.ly/2ZTh0eR

Busso, G. (2001). Vulnerabilidad social: nociones e implicancias de políticas para latinoamerica a inicios del siglo XXI. Seminario Internacional Las Diferentes Expresiones de La Vulnerabilidad Social En América Latina y El Caribe, 39. Recuperado de https://bit.ly/2J5K1gp

Canal Institucional, \& RTVC. (2017). Página Web Canal Institucional Tv. Recuperado de https://bit.ly/1K6OQBR Canal Uno. (2017). Página Web Canal Uno. Recuperado de https://bit.ly/1OGQt9p

Castillo, M., Camargo-Abello, M., \& Morales, R. (2011). Decisiones Acertadas de Educación en Emergencias. Bogotá: MEN-RET-UNICEF. Recuperado de https://bit.ly/2XG7hLv

Castro, A., Vizcaino, J., \& Alarcón Párraga, C. L. (2012). Atención Integral: Prosperidad para la Primera Infancia "De Cero A Siempre." Colombia, Colombia. Recuperado de https://bit.ly/1hgyKrT

Chaves Guimaraes, J. A., Batista Ernesto de Moraes, J., \& Duarte Moreira Guarido, M. (2007). Análisis documental de contenido de textos narrativos: bases epistemológicas y perspectivas metodológicas. Ibersid: Revista de Sistemas de Información y Documentación, 93-99. Recuperado de https://bit.ly/2REhdzp

CIESPAL. (2016). Página Web Centro Internacional de Estudios Superiores de Comunicación para América Latina (CIESPAL). Recuperado de https://bit.ly/2IMOUMx

Conde Gutiérrez, F. (2002). Encuentros y desencuentros entre la perspectiva cualitativa y la cuantitativa en la historia de la medicina. Revista Espanola de Salud Publica, 76(5), 395-408. https://doi.org/10.1590/S1135-57272002000500003 Congreso de Colombia. (2000). Ley 581 de 2000. Diario Oficial No. 44.026 Del 31 de Mayo de 2000. Recuperado de https://bit.ly/1GGpdl1 
Congreso de Colombia. Ley No. 1379 por la cual se organiza la Red Nacional de Bibliotecas Públicas y se dictan otras disposiciones (2010). Recuperado de https://bit.ly/1SjKXg8

Conpes-109. Política Pública Nacional de Primera Infancia. "COLOMBIA POR LA PRIMERA INFANCIA” (2007).

Colombia. Recuperado de https://bit.ly/2fSo5dh

Conpes-166. Politica Publica Nacional de discapacidad e inclusión social (2013). Colombia. Recuperado de

https://bit.ly/2DGGLoU

Contraloría General de la República de Colombia. (2014). Resultado de la evaluación a la política pública integral de desarrollo y protección social Estrategia de Atención Integral a la Primera Infancia De cero a Siempre 2010-2014. Bogotá: Imprenta Nacional de Colombia. Recuperado de https://bit.ly/2X5pL3A

Culver, S. H., \& Jacobson, T. (2012). Alfabetización mediática como método para fomentar la participación cívica. Comunicar, 20(39), 73-80. https://doi.org/10.3916/C39-2012-02-07

Delgado-Ponce, Á. (2015). La competencia comunicativa de los jóvenes en la sociedad líquida. Evaluación de la competencia mediática en adolescentes. Tesis doctoral: Universidad de Huelva. Recuperado de https://bit.ly/2Fxmbcj Escobar, A. (2005). Más allá del Tercer Mundo. Globalización y Diferencia. (ICANH - UNICAUCA, Ed.), ICANH UNICAUCA (ICANH-UN). Colombia: ICANH - UNICAUCA. Recuperado de https://unc.live/2ZMRp7a

Ferrés, J. \& Piscitelli, A. (2012). La competencia mediática: propuesta articulada de dimensiones e indicadores. Comunicar, XIX(38), 75-82. https://doi.org/10.3916/C38-2012-02-08

Freire, P. (1970). Pedagogía del oprimido. España: Siglo XXI editores. https://doi.org/10.3163/1536-5050.98.2.021 Freire, P. (1997). Pedagogía de la autonomía. Saberes necesarios para la práctica educativa. Educación y Territorio. Buenos Aires: Siglo XXI. Recuperado de https://bit.ly/2XG1XEX

Freire, P. (2004). Pedagogía de la Autonomía. Sao Pablo: Paz e Terra S.A. Recuperado de https://bit.ly/1jAZ2nF Fundación Acción Cultural Popular. (2013). Documental: La ignorancia es un pecado (Parte 1 de 3). Colombia: Banco de la República, Biblioteca Luis Ángel Arango. Recuperado de https://bit.ly/2J8xVDd

Fundación Nacional Batuta. (2017). Página Web Fundación Batuta. Recuperado de https://bit.ly/2Neowzq

García-Galera, C., \& Valdivia, A. (2014). Prosumidores mediáticos Cultura participativa de las audiencias y responsabilidad de los medios. Comunicar, 22(43), 10-13. https://doi.org/10.3916/C43-2014-a2

García-Ruiz, R., Ramírez-García, A., \& Rodríguez- Rosel, M. M. (2014). Educación en alfabetización mediática para una nueva ciudadanía prosumidora. Comunicar, 22(43), 15-23. https://doi.org/10.3916/C43-2014-01

García Sánchez, A. (2015). Contraespacios de re-existencia afrodescendiente. Wamom, Revista Dos Alunos Do Programa de PósGraduaçao Em Antropologia Social Da UFAM, 1(1), 29-42. Recuperado de https://bit.ly/2YaYAFD Gómez López, A. (2014). Putumayo: la vorágine de las caucherías. Memoria y testimonio. Primera parte. Bogotá: Centro Nacional de Memoria Histórica (CNMH). Recuperado de https://bit.ly/2ZVU4f1

ICBF, \& PNUD. (2013). ABC de la política pública de primera infancia, infancia y adolescencia. Colombia: Impresol Ediciones Ltada., Ed. Recuperado de https://bit.ly/1RZy1wq

Iniciativa de Red de Comunicación. (2004). Red de Comunicación e Información Juvenil, RIJ - Colombia. Recuperado de https://bit.ly/2ZKtQvG

Instituto Colombiano de Bienestar Familiar. (2016). Cómo hacer acompañamiento a los niños en el uso seguro de las tecnologías. Colombia. Recuperado de https://bit.ly/2J5DuSP

Instituto Colombiano de Bienestar Familiar. (2017). Página Web Instituto Colombiano de Bienestar Familiar. Recuperado de https://bit.ly/1iT6tow

Kaplún, M. (1985). El comunicador popular. Quito: CIESPAL-CESAP-RADIO NEDERLAND. Recuperado de https://bit.ly/2LvPDDI

Kaplún, M. (1990). Comunicación entre Grupos. El método del Cassette-Foro. Buenos Aires: E. Humanitas. Recuperado de https://bit.ly/2Xa5DgB

Kaplún, M. (1997). De medios y fines en comunicación educativa. Chasqui. 58, 4-6.

https://doi.org/http://dx.doi.org/10.16921/chasqui.v0i58.1120.g1149

Kaplún, M. (1998). Procesos educativos y canales de comunicación. Chasqui, 64, 4-8.

https://doi.org/10.1017/CBO9781107415324.004

López, G. (2012). Televisión Educativa. Colombia: Universidad del Bosque. Recuperado de https://bit.ly/2xaezrU

Lugo, M. (2010). Las políticas TIC en la educación de América Latina. Tendencias y experiencias. Revista Fuentes, 10, 52-68. Recuperado de https://bit.ly/2FvcQSE

Martin-Barbero, J. (2014). Pensar la Comunicación en Latinoamérica. Revista de Estudios Para El Desarrollo Social de La Comunicación, 10, 20-39. https://doi.org/10.15213/redes.n10.p21

Martín-Barbero, J. (1991). De los medios a las mediaciones. Comunicación, cultura y hegemonía. México: Ediciones G. Gill. Recuperado de https://bit.ly/322glcB

Martín-Barbero, J. (1996). Heredando el futuro. Pensar la educación desde la comunicación. Nómadas, 5, 2-14.

Recuperado de https://bit.ly/2Qjmdcl

Martín-Barbero, J. (2002). La educación desde la comunicación. Bogotá: Norma. Recuperado de https://bit.ly/2tqvXqs Mata, M. (1993). ¿Radio popular o comunitaria? Chasqui, 47. Recuperado de https://bit.ly/2IQDUO3

MEN. (2013). Competencias Para el Desarrollo Profesional TIC Docente. Bogotá : MinEducación - Colombia Aprende. Recuperado de https://bit.ly/1fjQBvf

MinCultura. (2013). Portafolio oferta institucional MinCultura. Bogotá. Recuperado de https://bit.ly/2LheYkn 
Ministerio de Educación Nacional de Colombia. (2017). Página Web Ministerio de Educación Nacional. Recuperado de https://bit.ly/2pGoMqP

Ministerio de la Protección Social. Política Nacional de Envejecimiento y Vejez 2007 - 2019 (2007). Colombia.

Recuperado de https://bit.ly/2KDAccM

Ministerio de Salud y Protección Social. (2017). Página web del Ministerio de Salud. Recuperado de https://bit.ly/2J6jkbn

MinTIC. (2016a). Estrategia de Gobierno en línea. Recuperado de https://bit.ly/2us6o6P

MinTIC. (2016b). Ministerio de las TIC. Recuperado de https://bit.ly/2Nh56d2

MinTIC. (2017). Plan Vive Digital - MinTIC. Recuperado de https://bit.ly/1rYQIZo

MinTIC. (2018a). En TIC confío. Recuperado de https://bit.ly/2J0mU8g

MinTIC. (2018b). SmartFilms y Cine Para Todos lanzan su categoría SmarTIC Incluyente para el 2018. Recuperado de https://bit.ly/2V153kL

Morin, E., Roger-Ciurana, E., \& Domingo-Motta, R. (2002). Educar en la era planetaria: el pensamiento complejo como mérito de aprendizaje en el error y la incertidumbre humana. Valladolid: UNESCO - Universidad de Valladolid.

MSPS - PDSP. (2013). Plan Decenal de Salud Pública, PDSP, 2012 - 2021. Ministerio de salud y Protección Socia.

Colombia. Recuperado de https://bit.ly/1z2XcAT

Muñoz-Borja, P. (2006). Construcción de sentidos del mundo de la discapacidad y de la persona con discapacidad.

Estudio de casos. Cali: Editorial de la Universidad del Valle.

Osorio Marulanda, C. (2011). El movimiento indígena colombiano: de la identidad negativa a la identidad positiva. El Ágora USB, 11, 49-65. Recuperado de https://bit.ly/2X4gxoj

Pedraza Gómez, Z. (2012). La disposición del gobierno de la vida: acercamiento a la práctica biopolítica en Colombia. Revista de Estudios Sociales, (43), 94-107. https://doi.org/10.7440/res43.2012.08

Pizarro, R. (2001). La vulnerabilidad social y sus desafíos: una mirada desde América Latina. Santiago de Chile: CEPA Recuperado de https://bit.ly/2IMfwxd

Prosperidad Social Colombia. (2016). Más Familias en Acción Mas compromiso por la Equidad. Recuperado de https://bit.ly/2Nb5eLd

Rincón, O., Saffon, M., Cadavid, A., Villegas, A., Ramírez, N., Martínez, M., El'Gazi, J. (2007). Ya no es posible el silencio, textos, experiencias y procesos de comunicación ciudadana. Bogotá: Centro de Competencia en Comunicación para América Latina - Friedrich Ebert Stiftung. Recuperado de https://bit.ly/2RzWay4

RTVC, \& Señal-Memoria. (2015). El Bachillerato por radio : la educación a distancia. Recuperado de

https://bit.ly/2xk1zzL

Scott, J. (1985). Weapons of the weak. Everyday forms of peasant resistance. New Haven and London: Yale University. https://doi.org/10.2307/2070255

Scott, J. (1986). Everyday Froms of Peasant Resistance. Journal of Peasant Studies, 13, 5-35.

https://doi.org/10.1111/j.1469-0691.2011.03558.x/pdf

Sen, A. (1999). Desarrollo y Libertad. Barcelona: Planeta.

SENA-MinTrabajo. (2013). Página oficial del Servicio Nacional de Aprendizaje, SENA. Recuperado de https://bit.ly/1flb57K

Señal Colombia. (2017). Página Web Señal Colombia. Recuperado de https://bit.ly/1fiEYCw

SNARIV. (2011). Enfoque diferencial. Unidad para la Atención y Reparación Integral a las Víctimas. Bogotá. Recuperado de https://bit.ly/2Wq0Siv

Teodoro Ramírez, M. (2007). Estadios de la otredad en la reflexión filosófica de Luis Villoro. Diánoia, LI/(58), $143-175$.

Recuperado de https://bit.ly/2xeYzVw

Tunubalá, T., \& Pechené-Muelas, M. (2010). "518 años de resistencia, 200 años de lucha de los pueblos”. El deber, el derecho de re-existencia y la libertad. Maguaré, (24). Recuperado de https://bit.ly/2IPJTTp

Ullmann, H., Maldonado Varela, C., \& Rico, M. N. (2014). La evolución de las estructuras familiares en América Latina, 1990-2010. Los retos de la pobreza, la vulnerabilidad y el cuidado. Santiago de Chile: CEPAL - UNICEF. Recuperado de https://bit.ly/2MC6Du9

Urna de Cristal Gobierno de Colombia. (2013). Todo lo que debes saber sobre el proyecto de vivienda gratis.

Recuperado de https://bit.ly/1wCqN4N

Urteaga, E. (2009). Las políticas de discriminación positiva. Revista de Estudios Politicos, (146), 181-213. Recuperado de https://bit.ly/2X3AFXt

Walsh, C. (2015). ¿Comunicación, decolonización y buen vivir? Notas para enredar, preguntar, sembrar y caminar (Vol. 1). https://doi.org/10.1017/CBO9781107415324.004 